ISSN 2525-5215

DOI: $10.48017 /$ dj.v6i4.1966

Volume 6, Número 4 (out./dez. 2021 ) pp: 4086-4106.

https://periodicos.ifal.edu.br/diversitas_journal/

\title{
C) Diversitas Journal \\ A Matemática Visual para o Desenvolvimento de Mentalidades Matemáticas em Alunos de uma Escola Pública
}

\section{Visual Mathematics for the Development of Mathematical Mentalities in Students of a Public School}

\author{
Fabrício de Oliveira Lima ${ }^{(1)}$; Daniel Nicolau Brandão $o^{(2)}$; \\ Douglas Muller da Silva ${ }^{(3)}$; Leonardo Rodrigues de Brito ${ }^{(4)}$; \\ Weverton de Barros Vieira ${ }^{(5)}$;
}

\author{
(1)ORCID: https://orcid.org/Oooo-0003-2224-0497; UNEAL/Graduando em Matemática, BRAZIL, E-mail: \\ fabriciollima98@gmail.com; \\ (2)ORCID: https://orcid.org/o0o0-0002-0291-8638; UNEAL/Professor Assistente, BRAZIL, E-mail: daniel.brandao@uneal.edu.br; \\ (s)ORCID: https://orcid.org/OOOO-0003-4387-8422; UNEAL/Graduando em Matemática, BRAZIL, E-mail: \\ prof.douglasmiller@gmail.com; \\ (4)ORCID: https://orcid.org/OOo0-0002-7915-9510; UNEAL/Graduando em Matemática, BRAZIL, E-mail: \\ leonardorodriguesrastellibrito@gmail.com;
}

(5)ORCID: https://orcid.org/o000-0003-2865-4084; UNEAL/Graduando em Matemática, BRAZIL, E-mail: wev2709@gmail.com;

Todo o conteúdo expresso neste artigo é de inteira responsabilidade dos seus autores.

Recebido em: 31 de julho de 2021; Aceito em: 29 de setembro de 2021; publicado em 19 de outubro de 2021. Copyright (C) Autor, 2021.

RESUMO: A presente pesquisa teve como objetivo desenvolver o senso numérico, através do uso de matemática visual, embasada na Teorias das Mentalidades Matemáticas em alunos do $9^{\circ}$ ano do ensino fundamental II de uma escola municipal do município de Junqueiro - AL. Estudos recentes na neurociência mostram que todos somos capazes de aprender matemática em alto nível desde que sejam estimulados de forma correta. É bem comum vermos alunos do ensino fundamental ao ensino superior afirmando que não gostam de matemática, pois bem, o motivo desse desprezo em matemática, na maioria das vezes é devido a forma que os conteúdos são ensinados, fazendo com que muitos alunos percam o interesse nessa área. Porém, existem outros métodos de ensinar matemática e um deles é a utilização da matemática visual. Diversos estudos mostram que trabalhar com a matemática visual faz com que o aluno ganhe autoconfiança, pois bem, o propósito desse trabalho é mostrar que a matemática visual pode levar o aluno a compreender de uma maneira mais clara o ensino da matemática. Ao decorrer desse trabalho compreenderemos, por meio de uma pesquisa de enfoque misto, como as abordagens de conteúdos por meio da matemática visual pode interferir no desenvolvimento cognitivo e aprendizagem em matemática nos alunos de uma escola pública no interior de Alagoas. Abordaremos conceitos e apresentaremos o resultado de um estudo de caso, tomando como base ideias presentes no livro da autora Britânica Jo Boaler, observando assim como esta metodologia pode ser transformadora. Analisando os dados da pesquisa, concluímos que a matemática visual realmente facilitou a aprendizagem dos alunos, bem como mudou a sua relação com a Matemática.

PALAVRAS-CHAVE: Neurociência. Matemática Visual. Ensino de matemática. Mentalidades Matemáticas.

ABSTRACT: The present research aimed to develop numerical sense, through the use of visual mathematics, based on the Theories of Mathematical Mentalities in 9th grade students of elementary school II of a municipal school in the city of Junqueiro - AL. Recent studies in neuroscience show that we are all capable of learning math at a high level as long as we are properly stimulated. It is quite common to see students from elementary school to higher education stating that they do not like math, well, the reason for this contempt in math, most of the time, is due to the way the contents are taught, causing many students to lose interest in that area. However, there are other methods of teaching mathematics and one of them is the use of visual mathematics. Several studies show that working with visual mathematics makes the student gain self-confidence, well, the purpose of this work is to show that visual mathematics can lead the student to understand the teaching of mathematics in a clearer way. In the course of this work, we will understand, through a research with a mixed focus, how content approaches through visual mathematics can interfere with cognitive development and learning in mathematics in students at a public school in the interior of Alagoas. We will discuss concepts and present the result of a case study, based on ideas present in the book by British author Jo Boaler, observing how this methodology can be transformative. Analyzing the survey data, we conclude that visual mathematics really facilitated students' learning, as well as changing their relationship with mathematics.

KEYWORDS: Neuroscience, Visual Mathematics, Teaching math, Mathematical Mentalities. 
LIMA, Fabricio de Oliveira; BRANDÃO, Daniel Nicolau; SILVA, Douglas Muller; BRITO, Leonardo Rodrigues de; VIEIRA, Weverton de Barros

INTRODUÇÃO

Ainda hoje, mesmo com tantas pesquisas na área da educação, o ensino de matemática, para algumas pessoas, resume-se apenas a cálculos e métodos fechados para resolver problemas que, muitas das vezes não fazem nenhum sentido para quem está aprendendo. Disto, surge o anseio em desenvolver um trabalho que aprimore as práticas de ensino-aprendizagem e possa ajudar na efetivação da melhora da educação matemática. Há quem diga que só pessoas inteligentes conseguem aprender matemática, ou que algumas nasceram com a capacidade de aprender matemática e outras não. Mas o problema pode está não na capacidade do aluno, mas na forma como a Matemática é abordada em sala de aula, pois infelizmente ainda hoje uma grande parte dos professores de matemática ainda trabalham o método mecanizado e não se permitem conhecer e aplicar novos métodos e estratégias de ensino.

A neurociência nos diz que nosso cérebro sempre está disponível para aprender, mas para isso é necessário que ele reconheça que o que está sendo posto a sua frente é significante e a melhor forma de capturar a atenção (a parte mais importante do processo ensino aprendizagem) é apresentar o conteúdo a ser estudado de maneira que os alunos o reconheçam como importante (Cosenza \& Guerra, 2011). Logo é necessário o desenvolvimento de uma prática transformadora, capaz de apresentar aos estudantes o quão interessante pode ser a matemática e a partir disso, construir um pensamento matemático baseado nas recentes descobertas da neurociência de como o cérebro aprende, foi desenvolvida a Teoria das Mentalidades Matemáticas que busca uma abordagem ativa do conhecimento de matemática, na qual os estudantes veem seu papel como o de compreensão e busca de sentido. Matemática é muito mais que apenas um amontoado de regras e métodos (BOALER, 2018).

Desenvolver o pensamento matemático ou uma mentalidade matemática, parte do desenvolvimento do Senso Numérico que, de acordo com Oliveira \& Basniak (2021, p. 5)

envolve a habilidade de aproximar e julgar o tamanho dos números, o que inclui o conhecimento sobre os números, suas operações e como utilizar esses conhecimentos para resolver problemas e calcular, além da escolha da representação numérica mais adequada dentro de um contexto e/ou para resolver problemas. 
LIMA, Fabricio de Oliveira; BRANDÃO, Daniel Nicolau; SILVA, Douglas Muller; BRITO, Leonardo Rodrigues de; VIEIRA, Weverton de Barros

Entre as várias abordagens apresentadas pela Teoria das Mentalidades Matemáticas, uma forma de abordar a Matemática de modo a fazer sentido para os alunos, e que ele consiga desenvolver o senso numérico, é utilização de componentes visuais nas aulas tais como material dourado, dobraduras, jogos e softwares matemáticos, o que é denominado de Matemática Visual. Segundo Park, J., \& Brannon, E. (2013), quando não pedimos aos alunos que pensem visualmente, perdemos uma incrível oportunidade de aumentar a compreensão deles, e de possibilitar um importante cruzamento cerebral, ou seja, compreender matemática é de extrema necessidade para o aperfeiçoamento do saber, porém essa compreensão somente se concretiza de forma mais eficaz quando aliada ao pensamento visual.

Pode-se afirmar que utilização de componentes visuais é extremamente útil para o ensino de matemática, pois descobertas importantes das neurociências sobre a utilização da matemática visual, falam que

\begin{abstract}
Nos últimos anos, os cientistas desenvolveram uma compreensão mais sutil das maneiras como nossos cérebros funcionam quando estudamos e aprendemos matemática. Nossos cérebros são compostos de "redes distribuídas", e quando lidamos com conhecimento, diferentes áreas do cérebro se iluminam e se comunicam. Quando trabalhamos em matemática, em particular, a atividade cerebral está espalhada por uma rede amplamente distribuída, que inclui duas vias visuais: as vias visuais ventral e dorsal. A neuroimagem mostrou que, mesmo quando as pessoas trabalham em um cálculo numérico, como $12 \times 25$, com dígitos simbólicos (12 e 25), nosso pensamento matemático é fundamentado no processamento visual. (BOALER, 2016, p.1)
\end{abstract}

Utilizando-se dessas informações, o objetivo da pesquisa foi desenvolver o senso numérico, através do uso de matemática visual, embasada na Teorias das Mentalidades Matemáticas em alunos do $9^{\circ}$ ano do ensino fundamental II de uma escola municipal do município de Junqueiro - AL.

\title{
A MATEMÁtica E A NEUROCIÊNCIA
}

A Base Nacional Comum Curricular (BNCC) (2018) diz que a matemática "é uma ciência humana, fruto das necessidades e preocupações de diferentes culturas, em diferentes momentos históricos, e é uma ciência viva, que contribui para solucionar problemas científicos e tecnológicos” (p. 267). É natural então que nas últimas décadas 
LIMA, Fabricio de Oliveira; BRANDÃO, Daniel Nicolau; SILVA, Douglas Muller; BRITO, Leonardo Rodrigues de; VIEIRA, Weverton de Barros

ela entre em um processo de transformação de uma forma significativa. Com o avanço da tecnologia, novas metodologias de ensino se tornaram possíveis em sala de aula. A tecnologia pode ser uma

ferramenta de auxílio à compreensão do raciocínio do aluno, de suas dificuldades e compreensões, além de ser uma poderosa ferramenta na elaboração de atividades que favoreçam a aprendizagem e até mesmo a individualização da aprendizagem, contribuindo com a autonomia do aluno (BITTAR, 2011 , p. 158).

Bittar (2011) conversa com as ideias de Bicudo (1985) que diz que, para o aluno compreender a realidade que está vivendo é necessário aumentar a sua confiança para enfrentar desafios e isso deve ser incorporado durante seu desenvolvimento na escola. Isso implica em aumentar as oportunidades para que os alunos possam ter mais ensejo de aprender. D’Ambrósio (1999) fala que "As práticas educativas se fundam na cultura, em estilos de aprendizagem e nas tradições" (p. 97). Entender como os alunos estão visualizando a educação é essencial e de fato precisamos mostrar aos estudantes que a matemática pode ser vista em diversos campos da vida humana e que a mesma faz parte da sociedade.

Segundo Parra e Saiz (1996), a realidade social vive em constante processo de transformação e que em determinados momentos da história humana estas tornam-se verdadeiras revoluções culminando em movimentos essenciais para a sociedade. $\mathrm{Na}$ educação, a organização dos conteúdos de ensino, e as metodologias dos docentes, tendem a mudar e à medida que a cultura vai sendo reconstruída se faz necessário adaptar metodologias e oferecer ao alunado novas formas de se aprender mais próximas do seu cotidiano. Além disso, cada estudante também é um indivíduo que está inserido na sociedade e suas vivências o acompanham à sala de aula. Ramos, Loresent e Petri (2016) falam que "as habilidades, experiências e conhecimentos prévios dos alunos tornam-se importantes fontes a serem valorizadas e exploradas pelos professores no planejamento e mediação pedagógica” (p. 2). Por a escola ser um espaço de reconstrução cultural, ela deve levar em conta o cotidiano de seus alunos e suas individualidades. Quando ela não cumpre o seu papel, acaba comprometendo as múltiplas inter-relações, que esse espaço encerra, com todo o contexto social.

É importante então no ensino de matemática que o aluno seja notado como um indivíduo que tem um processo de aprendizagem particular. Nessas perspectivas cabe entender um pouco sobre como a análise do funcionamento do cérebro (cognição) e sua 
LIMA, Fabricio de Oliveira; BRANDÃO, Daniel Nicolau; SILVA, Douglas Muller; BRITO, Leonardo Rodrigues de; VIEIRA, Weverton de Barros

relação com a aprendizagem podem ser benéficas para o ensino em matemática.

Entendemos que

A cognição é uma coleção de funções mentais que incluem, entre outros aspectos, a atenção, concentração, percepção, compreensão, aprendizagem, memória, resolução de problemas e raciocínio, que permitem que o indivíduo compreenda e se relacione com o mundo social e seus elementos. (PARENTÉ apud RAMOS, LORESENT E PETRI, 2016, p. 7)

Quando falamos no cognitivo a primeira indagação que surge, é como os estímulos são processados e transformados em informações. Esse estudo é, de fato, fundamental para a compreensão da matemática visual, pois, a mesma está estritamente ligada ao funcionamento e processamento de informações pelos neurônios. Se faz necessário partirmos de duas vertentes que podem auxiliar na compreensão do assunto, que seriam a neurociência cognitiva e a neuropsicologia.

Dentro do campo do estudo do cérebro, existem diferentes ramos, quando relacionamos esse estudo a educação, estamos falando da neurociência cognitiva (Temple, 1997), que não nega a natureza complexa e integrativa do cérebro-mente, mas postula que a análise das dissociações de desempenho em indivíduos com lesões ou disfunções cerebrais pode ser uma estratégia para identificar os componentes parcialmente autônomos a partir dos quais o sistema é construído, ou seja, a partir da detecção de estímulos produzidos pelo cérebro, é possível identificar uma metodologia que pode ajudar no desenvolvimento da aprendizagem. A neuropsicologia cognitiva analisa os diferentes padrões de processos psicológicos comprometidos e preservados em decorrência de formas distintas de lesões ou disfunções cerebrais com o intuito de identificar duplas-dissociações (Shallice, 1988).

Quando vamos para a análise desta na matemática, percebemos que nos últimos anos surgiram diversas pesquisas que procuram associar a matemática com as funções do cérebro. O cérebro possui diversas redes conectadas, onde muitas são ativadas durante a execução de atividades, como por exemplo quando o aluno faz determinadas tarefas. Isto é bem descrito por Menon, onde ele afirma que uma rede cerebral amplamente distribuída estabelece o processamento mental do conhecimento matemático (MENON, 2014). Algumas pesquisas, apresentadas por Jo Boaler (2008) mostram que quando o aluno é estimulado da forma correta e com recursos visuais eles atingem mais áreas do cérebro fazendo com que a aprendizagem aconteça com maior clareza, enquanto quando 
LIMA, Fabricio de Oliveira; BRANDÃO, Daniel Nicolau; SILVA, Douglas Muller; BRITO, Leonardo Rodrigues de; VIEIRA, Weverton de Barros

eles participam de aulas convencionais não se tem tantas áreas ativadas. Com isso temos bagagem suficiente para compreender o que é a matemática visual e como ela pode ser importante para a aprendizagem dos alunos.

\section{Senso numérico}

Para Corso e Dorneles (2010), o senso numérico é "a facilidade e a flexibilidade das crianças com números e a sua compreensão do significado dos números e ideias relacionadas a eles.” (p. 299). Esta é uma habilidade que pode ser aparentemente mais natural para alguns alunos, mas, por ser uma habilidade, pode ser desenvolvida ao longo da vida estudantil de todos os estudantes. O senso numérico inclui "consciência, intuição, reconhecimento, conhecimento, habilidade, desejo, sentimento, expectativa, processo, estrutura conceitual ou linha numérica mental.” (Berch apud Corso e Dornelles, 2010, p. 299). Ferrari apud Dahaene (2008) assume que o significado de senso numérico é "a capacidade inata de perceber diferenças de quantidades" (p. 53) como também a capacidade de reconhecer "a mudança de uma pequena coleção quando, sem seu conhecimento direto, se remove ou se acrescenta um objeto." (p. 53).

Dehaene apud Ferrari (2008) relata que as construções matemáticas são fruto atividade coerente acabada do cérebro. Assim, o senso numérico está estritamente ligado com as funções que o cérebro desenvolve. Além disso é uma habilidade cognitiva que possui inter-relação com o meio social que os estudantes estão inseridos. É importante que entendamos que o senso numérico é uma habilidade que não tem como ser ensinada como um conteúdo, porém, este deve ser desenvolvido ao longo do percurso escolar através de atividades que estimulem o cérebro.

Nesta perspectiva, ao conhecer a realidade que envolve os alunos, o professor pode entender até que ponto ele precisa desenvolver o senso numérico. $O$ senso numérico é muito importante para todos os alunos, pois com essa habilidade adquirida os alunos desenvolvem diferentes formas de raciocínio para a resolução de determinado problema fugindo de estratégias que recorrem a simples memorização. Corso e Dornelles (2010) alertam que "um senso pouco desenvolvido sobre o que os números representam torna uma tarefa do tipo aprender multiplicação um puro exercício de memorização.” (p. 300) 
LIMA, Fabricio de Oliveira; BRANDÃO, Daniel Nicolau; SILVA, Douglas Muller; BRITO, Leonardo Rodrigues de; VIEIRA, Weverton de Barros

O senso numérico, pode desenvolver nos alunos altos níveis de aprendizagem (FEIKES; SCHWINGENDORF, 2008). Percebemos que nas escolas brasileira alguns alunos não utilizam esta habilidade para pensar formas alternativas para a resolução de um determinado problema. Algo interessante é que ter o senso numérico não é sinônimo de ser o mais rápido durante a resolução de uma questão, nessa perspectiva o tempo não é tão importante, mais o caminho que o aluno percorre e onde eles chegam. Durante as aulas os professores precisam tomar muito cuidado, pois quando exigimos que os alunos apresentem as respostas memorizadas podemos desenvolver neles ansiedade matemática (BOALER, 2014).

Em algumas pesquisas com alunos, mostram que testes cronometrados e a memorização causam uma série de pressões, a ponto de os alunos desistirem e não continuar com prazer em aprender matemática. De fato, o senso numérico precisa ser visto como uma habilidade que precisa ser desenvolvida a cada ano escolar. É necessário que os professores conheçam sua importância e trabalhem em conjunto para desenvolver essa habilidade nos alunos.

\section{A matemática visual}

A matemática visual é uma parte da matemática que une o raciocínio voltado a precisão com a criatividade, onde o aluno é capaz de construir diferentes formas com valor lógico para se chegar a um resultado. Nesta, o aluno usa a imagem como recurso, além de materiais manipuláveis e interliga a matemática ao seu cotidiano. Segundo Novaes (2009), a "vasta possibilidade de exposições visuais, a visualização vem se destacando como área de interesse de muitos pesquisadores em educação matemática, que ressaltam sua importância na aprendizagem da matemática.” (p. 33).

A matemática visual vai além do uso somente de imagens, mas também se refere a usar jogos, vídeos, plataformas e atividades práticas, pois, quando impulsionamos os alunos a reconhecerem a matemática em seu dia-a-dia, eles entendem a necessidade em aprender. A matemática visual enfatiza o desenvolvimento do pensamento matemático, onde este pensamento matemático é denominado de mentalidade matemática. Segundo BOALER (2018,p. 35), “o cérebro só é capaz de comprimir conceitos; ele não é capaz de comprimir regras e métodos". Nesta perspectiva a autora entende que a matemática é 
LIMA, Fabricio de Oliveira; BRANDÃO, Daniel Nicolau; SILVA, Douglas Muller; BRITO, Leonardo Rodrigues de; VIEIRA, Weverton de Barros

muito mais que apenas um amontoado de regras e métodos, e isso é o que faz tantos alunos terem pavor da Matemática.

A matemática é fundamentada em diversas áreas em encontrar padrões e abstrações nas estruturas da realidade através de manipulação. Essa manipulação é denominada de matematização (NOVAES, 2009, p. 34). Ele ainda complementa que "percebemos algumas semelhanças nos objetos que nos levam à abstração do que é comum e submetemos esta percepção a uma elaboração racional e simbólica que nos permita manipular suas estruturas” (NOVAES, 2009, p. 34).

\begin{abstract}
Nossa percepção humana é fortemente visual e assim não é de se estranhar que o apoio contínuo ao aspecto visual esteja tão presente nas tarefas relacionadas à matematização, não só naquelas que, como a geometria, lidam mais diretamente com aspectos do espaço, mas também em algumas outras, como a análise matemática, que surgiu para explorar os diferentes tipos de mudanças que acontecem nos objetos materiais. Os matemáticos frequentemente usam processos simbólicos, diagramas, e muitas outras formas de processos mentais que envolvem a imaginação em seu trabalho. Eles os ajudam a adquirir o que poderíamos chamar de uma intuição do abstrato, um conjunto de reflexos mentais, algo como uma visão unitária das relações entre diferentes objetos. Deste modo eles parecem saber com antecedência como estes diferentes objetos vão reagir quando introduzirem algumas mudanças convenientes em alguma parte da estrutura. (NOVAES APUD GUZMÁN, 2009, p. 34)
\end{abstract}

Comumente em nossas escolas percebemos que muitos alunos são induzidos a memorizar, onde usar os dedos para contar, é visto como algo ruim. Porém, o estímulo visual causado pela visualização nos dedos é de extrema importância para a concretização da aprendizagem. Novaes (2009) diz que a visualização é um recurso que pode abrir um modo diferente de trabalhar o pensamento matemático, além do linguístico e axiomático das demonstrações tradicionais e da manipulação simbólica da álgebra. Se faz necessário que as escolas enfatizem a importância de usar os dedos para contar, pois isso sendo trabalhado desde pequeno o aluno não sentirá medo de usar os mesmos em turmas maiores. Brian Butterworth, renomado estudioso do cérebro nessa área, afirma que, se os alunos não estão aprendendo sobre os números por meio da reflexão sobre seus dedos, os números "jamais terão uma representação normal no cérebro” (BUTTERWORTH, 1999, p. 249-250). Em alguns lugares os alunos são repreendidos pelos pais e professores, e muitas das vezes, eles escondem as mãos para os professores não verem eles utilizando os dedos. Os neurocientistas recomendam que os 
LIMA, Fabricio de Oliveira; BRANDÃO, Daniel Nicolau; SILVA, Douglas Muller; BRITO, Leonardo Rodrigues de; VIEIRA, Weverton de Barros

dedos sejam vistos como uma ligação entre os números e sua representação, além de um suporte na resolução de problemas de aritmética.

Os alunos do século XXI corriqueiramente, estão submissos ao pensamento de que ser o primeiro a terminar é sinônimo de ser o melhor, e isso é um dos paradigmas que precisamos quebrar, para Jo Boaler (2018) muitas crianças que poderiam ter um ótimo futuro na matemática acabam desistindo por achar que não são rápidas o bastante. Precisamos mostrar aos nossos alunos que existem diferentes formas de se resolver um mesmo questionamento e que fazer é mais importante do que ser o primeiro a terminar. Outro grande problema que enfrentamos em nossa sala de aula é como o erro é visto, onde muitos alunos têm o pensamento que não deve errar, nós enquanto educadores devemos compreender que o erro é importante para que saibamos onde devemos intensificar a aprendizagem, quando cometemos erros e os corrigimos nosso cérebro aprende mais.

No Brasil o Instituto Sindarta possui um programa de atividades, criado em 2015, que visa aplicar as metodologias de pesquisa de Jo Boaler, Elizabeth Cohen e Rachel Lotan. Este visa disseminar as ideias e já alcançaram diversos resultados na evolução da aprendizagem dos alunos. As autoras acreditam que o trabalho em grupo e usando recursos visuais podem aumentar de maneira significativa os conhecimentos dos alunos. O cérebro humano é capaz de ser mudado no tamanho e aquisições de informações em curto prazo, existe neste uma área chamada hipocampo, ele é uma parte do cérebro que é especializada na aquisição e utilização de informações espaciais complexas, se o cérebro pode mudar em semanas, quando ensinado da forma correta durante o ano o aluno pode desenvolver imensa aprendizagem. O trabalho do instituto é notório em plataformas digitais, onde ofertam webinar e formações para atingir mais professor do Brasil. Enquanto papel da educação em transformar, devemos buscar sempre mudar a nossa realidade, transformar antes de tudo o ser humano e a matemática como uma ciência social deve ser apresentada como tal, enquanto educadores a mudança em nossa sala de aula começa com a nossa mudança pessoal, quando responsável pelo que cativamos durante nossa jornada. 
LIMA, Fabricio de Oliveira; BRANDÃO, Daniel Nicolau; SILVA, Douglas Muller; BRITO, Leonardo Rodrigues de; VIEIRA, Weverton de Barros

METODOLOGIA

Este estudo se constitui em uma pesquisa mista que de acordo com Sampieri, Collado e Lucio (2013, p. 550)

são a integração sistemática dos métodos quantitativo e qualitativo em um só estudo, cuja finalidade é obter uma "fotografia" mais completa do fenômeno. Eles podem ser unidos de tal forma que a abordagem quantitativa e a qualitativa conservem suas estruturas e procedimentos originais ("forma pura dos métodos mistos"). Esses métodos também podem ser adaptados ou sintetizados para realizar a pesquisa e driblar os custos do estudo.

Utilizamos um desenho não experimental e descritivo e de corte longitudinal, tendo uma amostra da pesquisa não probabilística e intencional, onde participaram 17 alunos do $9^{\mathrm{o}}$ ano do Ensino Fundamental da escola Escola Municipal de Educação Básica Mônica Rosa de Jesus. Como instrumento de pesquisa foram utilizados dois questionários com 17 alunos participantes, o primeiro questionário como pré-teste (Anexo I), contendo 13 questões com o objetivo de entender quais os conhecimentos que os alunos possuíam com os conteúdos matemáticos de divisão, frações, mínimo múltiplo comum e equação do segundo grau antes da aplicação do projeto e o segundo questionário contendo 12 questões no qual denominamos de pós-teste (Anexo II) foi aplicado ao final da intervenção.

\section{Procedimentos}

Como a pesquisa tinha como objetivo verificar se a matemática visual poderia desenvolver o senso numérico dos alunos, primeiramente foi feita uma pesquisa referente aos conteúdos de razões e frações, equações de segundo grau, multiplicação, divisão e máximo divisor comum. Feita a pesquisa teórica e a busca de metodologias para a prática e então ficou determinado que a equipe iria fazer a aplicação prática da pesquisa em uma turma de nono ano da Escola Municipal de Educação Básica Mônica Rosa de Jesus

Devido a pandemia vivida na época da pesquisa, a aplicação foi feita online por meio do aplicativo de reuniões Google Meet que também permite o compartilhamento de tela, além deste, também foi utilizado softwares como o Paint e o Word. 
VISUAL MATHEMATICS FOR THE DEVELOPMENT OF MATHEMATICAL MENTALITIES IN STUDENTS OF A PUBLIC SCHOOL

LIMA, Fabricio de Oliveira; BRANDÃO, Daniel Nicolau; SILVA, Douglas Muller; BRITO, Leonardo Rodrigues de; VIEIRA, Weverton de Barros

Multiplicação, Divisão e Máximo Divisor Comum

A seguir veremos como foi realizada a aplicação dos conteúdos e quais estratégias de matemática visual foram utilizadas. Na primeira aplicação foi mostrado aos alunos um método de contagem para realizar as multiplicações e divisões. É um método bem trivial, porém facilita aos alunos a enxergarem por meio da separação que uma multiplicação pode ser vista como uma série de repetidas somas de mesma parcela. No caso da divisão, dá uma noção visual do conceito de resto.

Figura 1 - Representação visual conceito de resto.
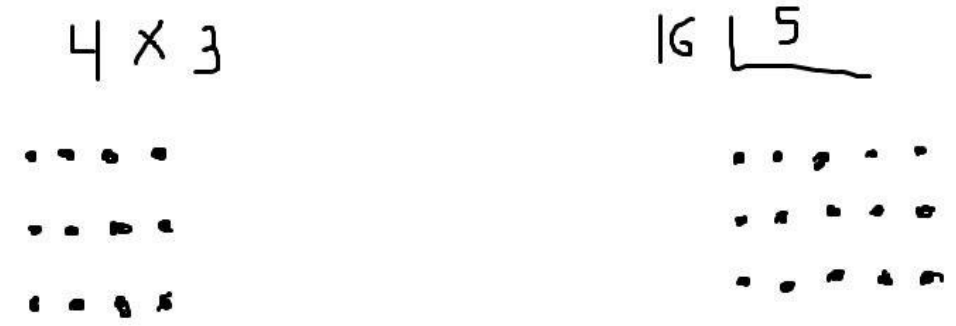

Fonte: Elaborado pelos pesquisadores

Em seguida foi apresentado um método de multiplicação através de desenhos de linhas. Trata-se de desenhar linhas paralelas que representam os fatores da multiplicação desejada e contar todos os pontos de interseção entre elas. Começou-se por multiplicações entre fatores de um dígito, mas também se avançou para multiplicações de fatores com mais dígitos.

Figura 2 - Multiplicação visual
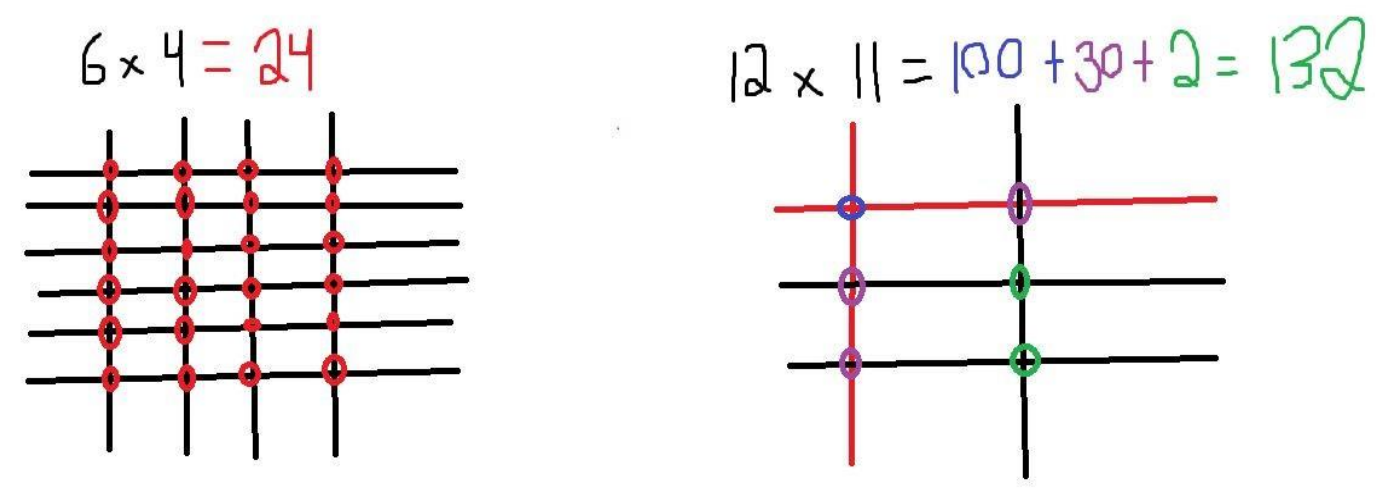

Fonte: Elaborado pelos pesquisadores 
VISUAL MATHEMATICS FOR THE DEVELOPMENT OF MATHEMATICAL MENTALITIES IN STUDENTS OF A PUBLIC SCHOOL

LIMA, Fabricio de Oliveira; BRANDÃO, Daniel Nicolau; SILVA, Douglas Muller; BRITO, Leonardo Rodrigues de; VIEIRA, Weverton de Barros

Na segunda aplicação, foi apresentado aos alunos o aplicativo para celular Simple Soroban. Este aplicativo é um ábaco japonês (Soroban) simples. Há um modo para usar o ábaco livremente, e há um modo "desafio" com quatro operações (Soma, Subtração, Multiplicação de 1 e 2 dígitos e Divisão) e três níveis de dificuldade (Fácil, Médio E Difícil). O aplicativo foi apresentado para a turma em seu funcionamento com os alunos verificando e participando ativamente da aplicação. $\mathrm{O}$ aplicativo permitiu a união da matemática visual com a gamificação e foi interessante notar que os alunos mesmo após as aulas continuaram a realizar os desafios do aplicativo.

Figura 3

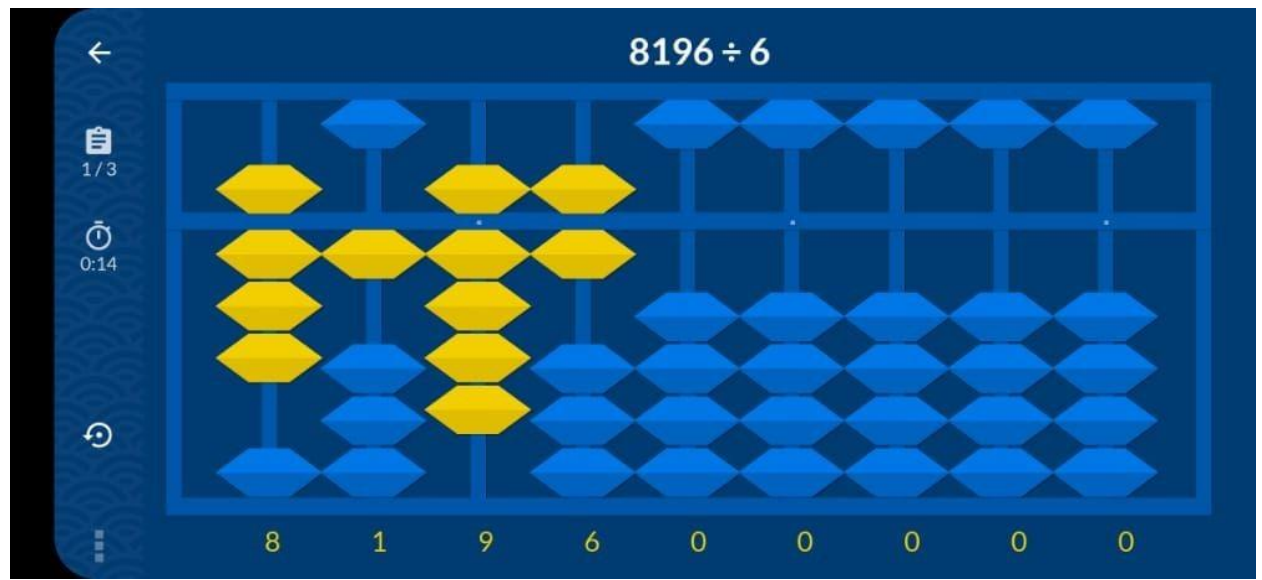

Fonte: Elaborado pelos pesquisadores

Na terceira aplicação foi feita uma apresentação sobre o conceito de Máximo Divisor Comum (MDC) e logo após uma forma visual de resolução de problemas objetivos envolvendo blocos de quadradinhos representando os números. Os divisores de cada número foram sendo preenchidos através da resposta da pergunta "De quantos em quantos quadradinhos podemos preencher as colunas de forma que não fique sobrando ou faltando nenhum?” Todas as possibilidades foram listadas. Em seguida, era verificado o maior número comum entre os números e assim, sinalizado o MDC. 
VISUAL MATHEMATICS FOR THE DEVELOPMENT OF MATHEMATICAL MENTALITIES IN STUDENTS OF A PUBLIC SCHOOL

LIMA, Fabricio de Oliveira; BRANDÃO, Daniel Nicolau; SILVA, Douglas Muller; BRITO, Leonardo Rodrigues de; VIEIRA, Weverton de Barros

Figura 4- Representação visual de Máximo Divisor Comum

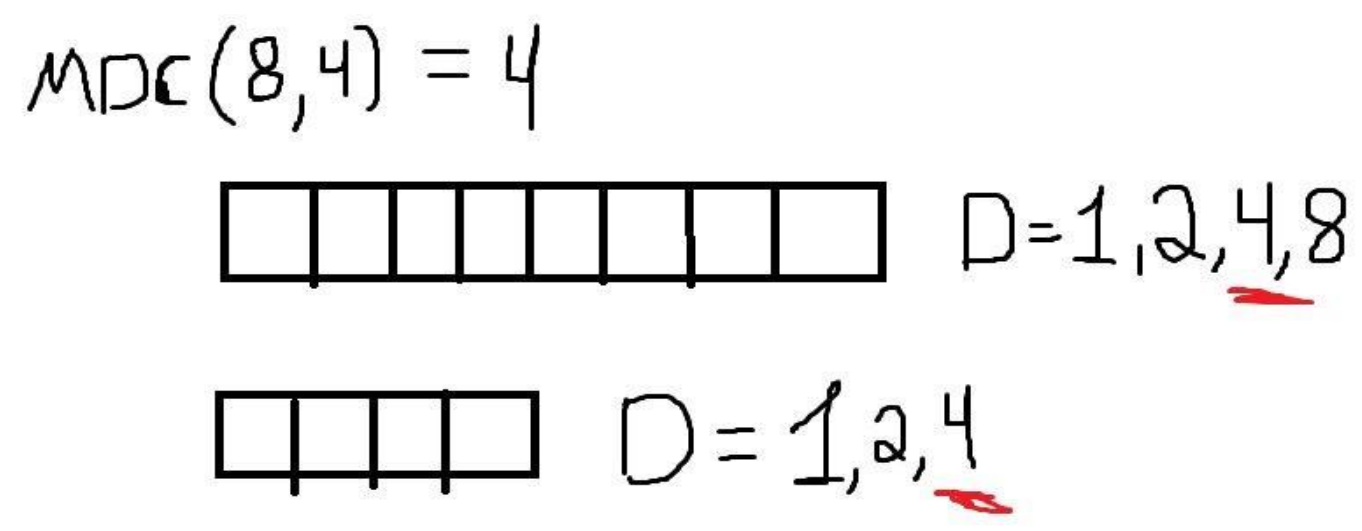

Página | 4098

Fonte: Elaborado pelos pesquisadores

$\mathrm{Na}$ quarta aplicação, a partir da noção concedida no encontro anterior, foi apresentado a noção visual do método das subtrações sucessivas para o entendimento de cálculo da turma. Esse método consiste em sempre subtrair o menor número do maior sucessivamente até que ambos os números sejam iguais; este número então, é o MDC.

Figura 5 - Método de subtrações sucessivas

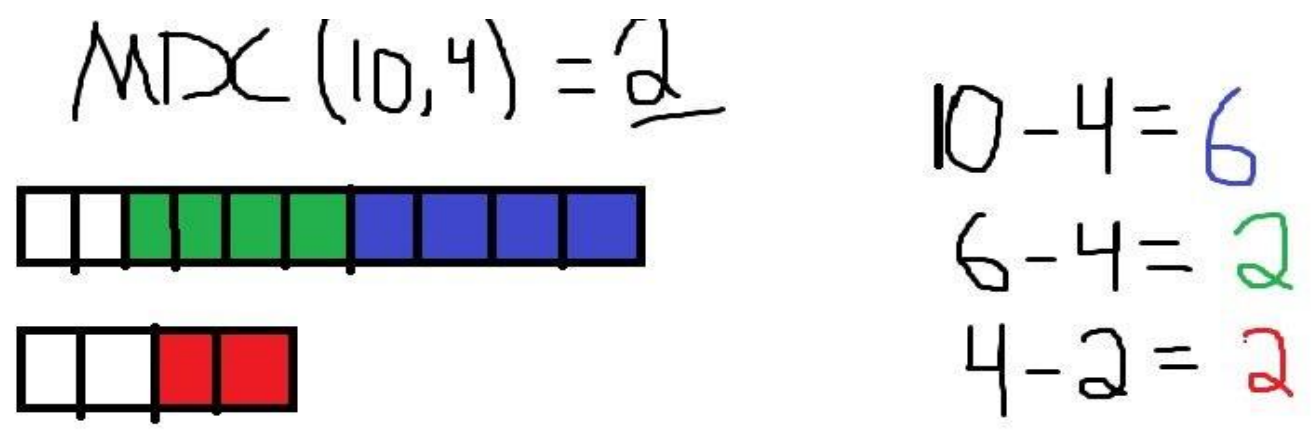

Fonte: Elaborado pelos pesquisadores

Após isso, foi proposto aos alunos problemas que envolviam o cálculo de MDC. Grande parte da turma utilizou do método das subtrações sucessivas e responderam corretamente às questões.

\section{Frações e Equação do Segundo Grau}

Nesta primeira aula, associamos as frações ao nosso cotidiano. Mostramos que elas são importantes para indicar partes. Falamos sobre frações, como elas se 
VISUAL MATHEMATICS FOR THE DEVELOPMENT OF MATHEMATICAL MENTALITIES IN STUDENTS OF A PUBLIC SCHOOL

LIMA, Fabricio de Oliveira; BRANDÃO, Daniel Nicolau; SILVA, Douglas Muller; BRITO, Leonardo Rodrigues de; VIEIRA, Weverton de Barros

comportam, os diferentes tipos e como calcular a fração de uma quantidade. Além disso apresentamos as operações com frações.

Figura 6 - Diferentes representações de frações
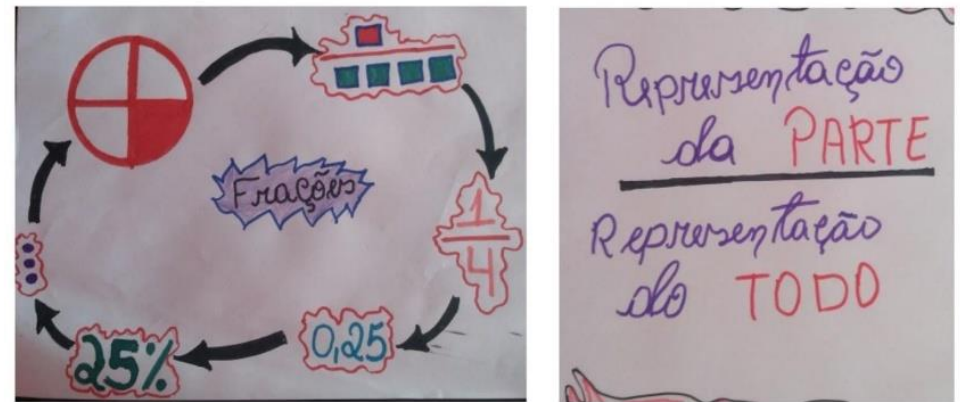

Fonte: Elaborado pelos pesquisadores

Durante as aulas usamos copos divididos em partes e colocamos $1 / 3$ de água em uma $2 / 3$ de água em outro, usamos um terceiro para mostrar que quando os denominadores são iguais basta somar a parte de cima e repetir o denominador. Como mostrado a seguir:

Figura 7 - Soma de frações com mesmo denominador
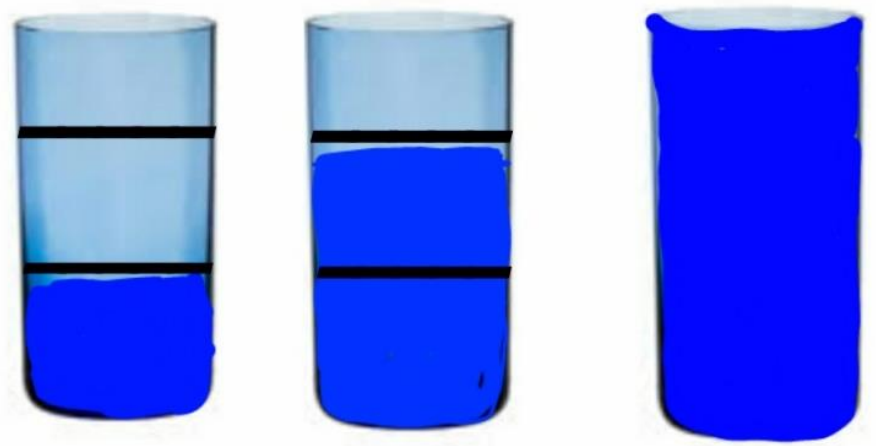

Fonte: Elaborado pelos pesquisadores

Mostrou-se como usar a matemática visual para melhorar a interpretação da questão e despertamos a criatividade. Apresentamos para eles as operações através dos métodos convencionais e também usamos regras para encontrar frações equivalentes. Como mostrado a seguir: 
VISUAL MATHEMATICS FOR THE DEVELOPMENT OF MATHEMATICAL MENTALITIES IN STUDENTS OF A PUBLIC SCHOOL

LIMA, Fabricio de Oliveira; BRANDÃO, Daniel Nicolau; SILVA, Douglas Muller; BRITO, Leonardo Rodrigues de; VIEIRA, Weverton de Barros

Figura 8 - Representação de operações usando diferentes métodos
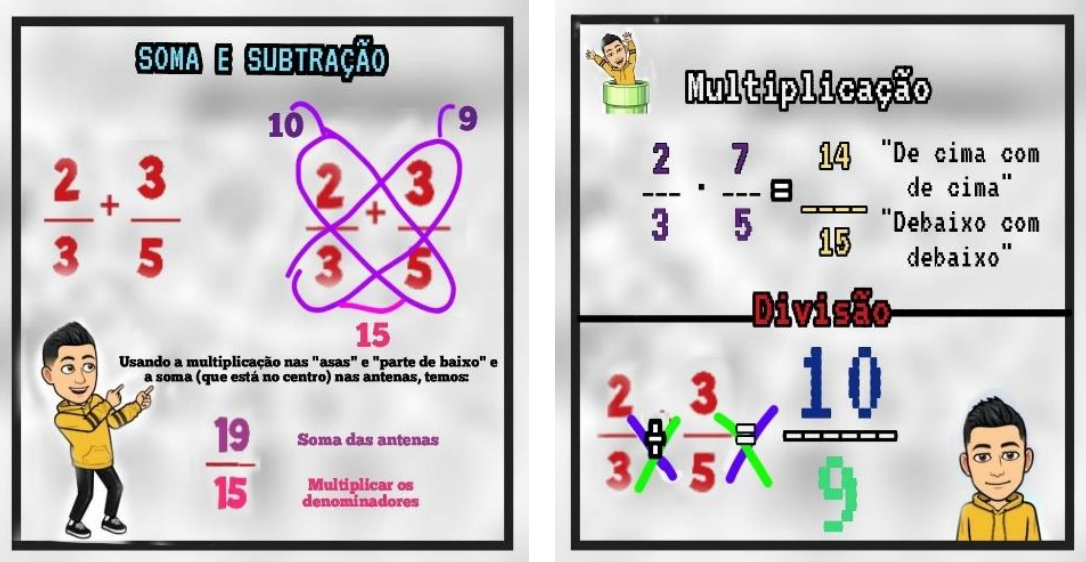

Página | 4100

Fonte: Elaborado pelos pesquisadores

Durante a segunda aula iniciamos o conteúdo equação do segundo grau. Inicialmente mostramos a importância e onde usar o conteúdo no cotidiano. Depois disso, fizemos a demonstração geométrica da fórmula atribuída a Bháskara. Como mostrado a seguir:

Figura 9 - Demonstração visual da fórmula de Bháskara
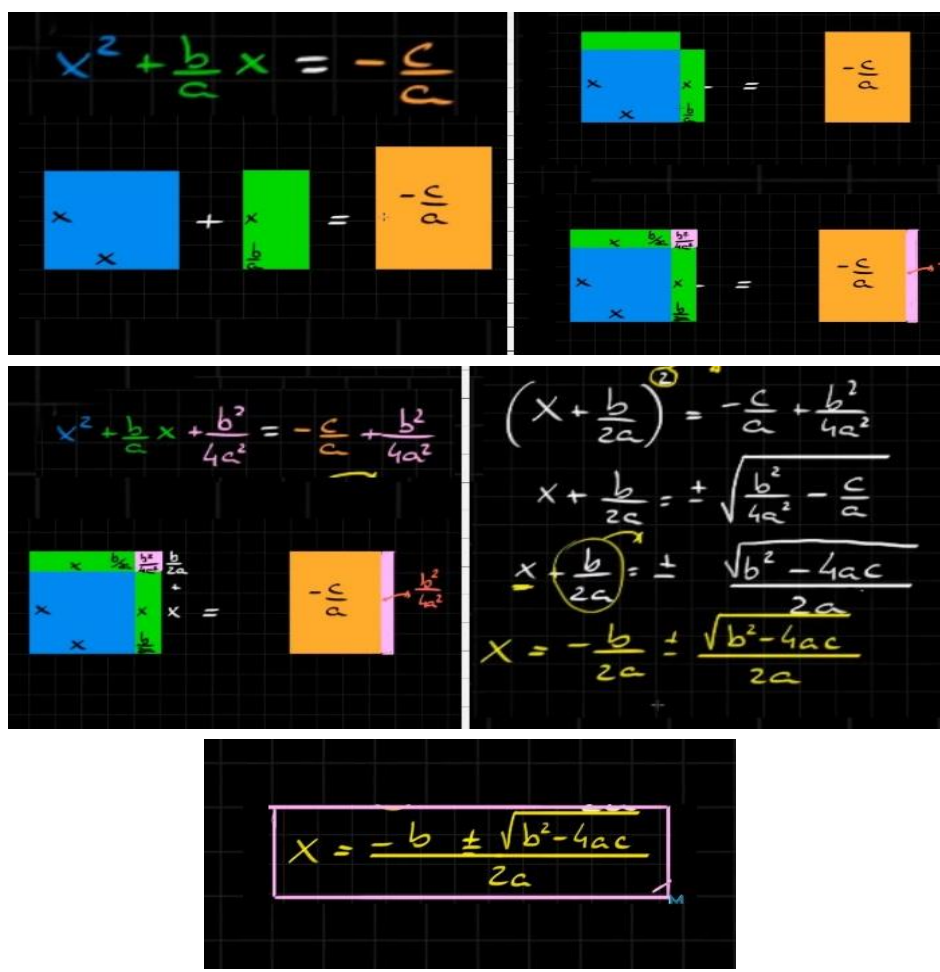

Fonte: Elaborado pelos pesquisadores (2020) 
VISUAL MATHEMATICS FOR THE DEVELOPMENT OF MATHEMATICAL MENTALITIES IN STUDENTS OF A PUBLIC SCHOOL

LIMA, Fabricio de Oliveira; BRANDÃO, Daniel Nicolau; SILVA, Douglas Muller; BRITO, Leonardo Rodrigues de; VIEIRA, Weverton de Barros

Durante a aula usamos apresentamos a ideia de coeficientes, os tipos de equação, completa e incompleta, e ensinamos a interpretar e resolver equações desse tipo. Usamos como exemplo a questão a seguir:

Figura 10 - Resolução usando os aspetos visuais
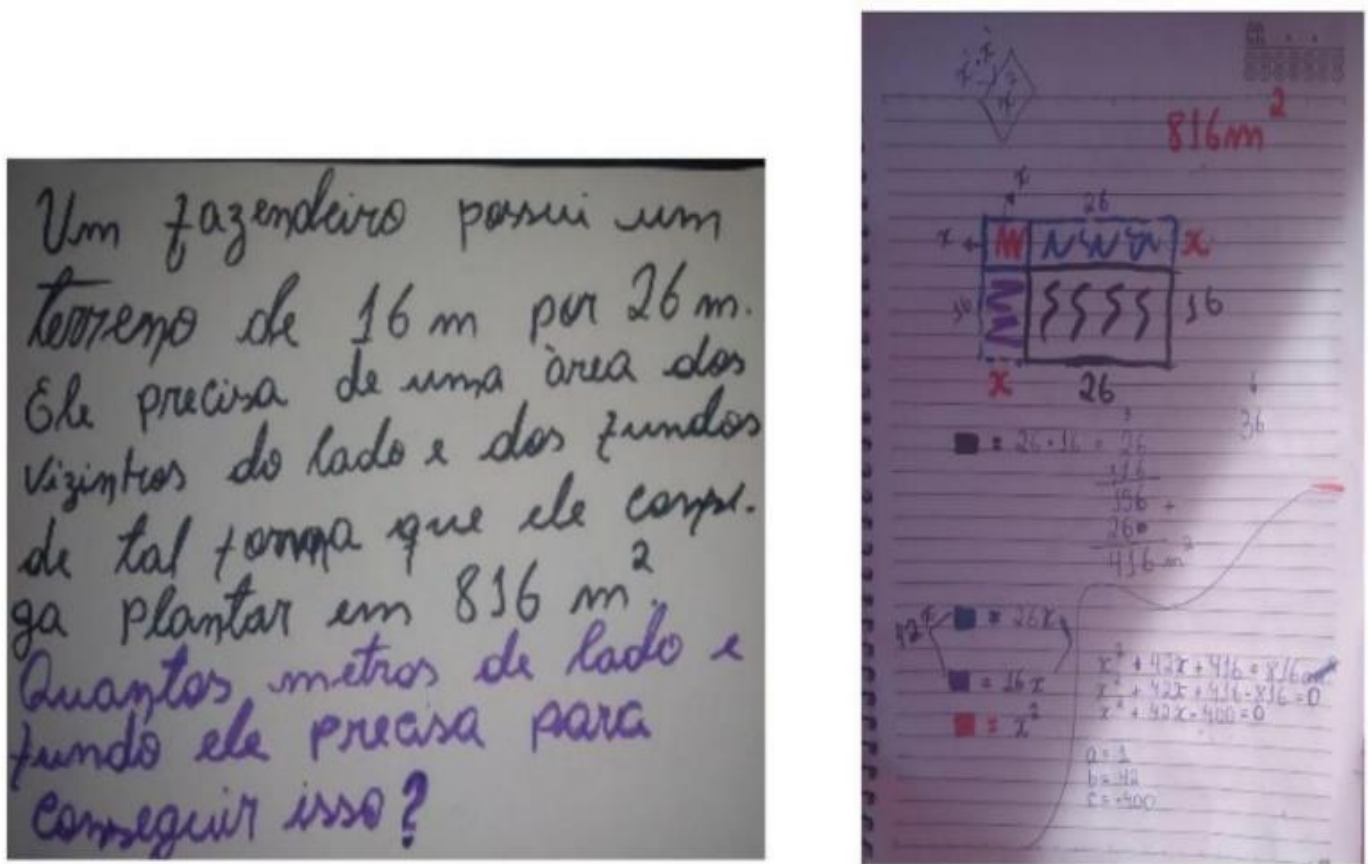

Fonte: Elaborado pelos pesquisadores

$\mathrm{Na}$ última aula apresentou-se o que era as equações incompletas e associamos ao teorema de Pitágoras. Onde fizemos diversas demonstrações visuais como mostrado a seguir: 
VISUAL MATHEMATICS FOR THE DEVELOPMENT OF MATHEMATICAL MENTALITIES IN STUDENTS OF A PUBLIC SCHOOL

LIMA, Fabricio de Oliveira; BRANDÃO, Daniel Nicolau; SILVA, Douglas Muller; BRITO, Leonardo Rodrigues de; VIEIRA, Weverton de Barros

Figura: 12 - Demonstração visual do teorema de Pitágoras

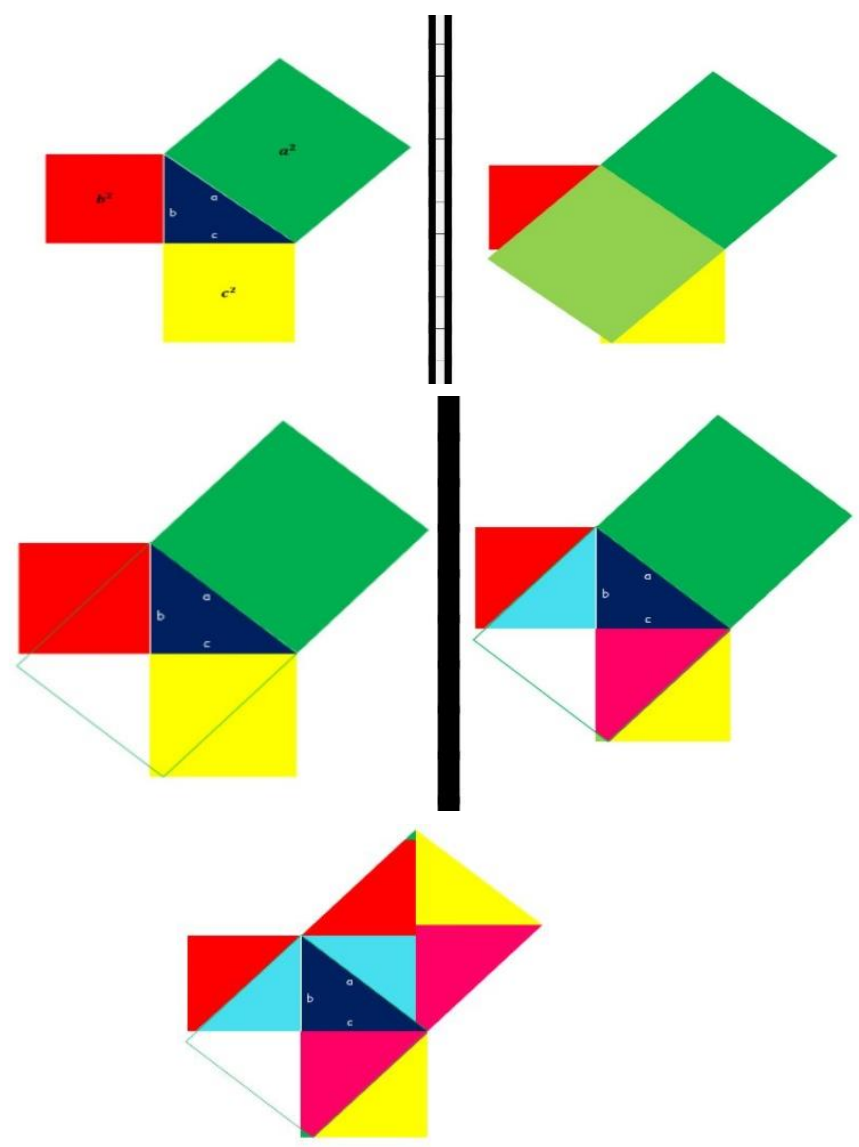

Página | 4102

Fonte: Elaborado pelos pesquisadores

\section{RESULTADOS E DISCUSSÕES}

Durante o trabalho procuramos entender como a matemática está interligada a utilização de recursos visuais e como estes contribuíram na aprendizagem dos alunos. Neste contexto, durante a pesquisa percebemos que a matemática visual não pode ser desvinculada da neuromatemática que estuda o comportamento do cérebro ao ser instigado com diferentes tipos de incentivos matemáticos. O processo de aprendizagem dos alunos aconteceu de forma significativa, à medida que as atividades foram desenvolvidas eles demostraram progresso e grande aceitação da metodologia. Ao realizar as atividades foi notório que mesmo tendo dificuldades, tendo em vista que o trabalho foi realizado de forma remota, eles demostram interesse em aprender os conceitos apresentados nas aulas. 
LIMA, Fabricio de Oliveira; BRANDÃO, Daniel Nicolau; SILVA, Douglas Muller; BRITO, Leonardo Rodrigues de; VIEIRA, Weverton de Barros

Analisando os dados referentes ao pré-teste, a média de acerto em questões de fração foi $52,95 \%$, m.m.c. 41,2\% e 62,74\% em questões de equação do segundo grau. A última pergunta do questionário, a qual tratou sobre a forma com que os professores ministram as aulas de Matemática e a visão de como deveria ser o ensino da matemática. O resultado foi o seguinte: $63 \%$ dos alunos afirmaram que não concordam com o modo que os professores ensinam, enquanto $37 \%$ dos alunos afirmaram que concordam.

Alguns alunos fizeram os seguintes comentários:

O ensino da matemática deveria usar de formas criativas e diferentes durantes as aulas.

A aprendizagem da matemática deve ser bem trabalhada para que proporcione ao aluno uma experiência articulada e promissora, com o objetivo de um ensino que sirva a inserção social proporcionando o desenvolvimento do seu potencial junto com o meio de interação, utilizando atividades diferentes, jogos e brincadeiras.

Um pequeno percentual (37\%) que aceitam as metodologias dos professores representam aqueles alunos que já tem afinidade para a Matemática. Segundo Carvalho (1994, p. 15) "a Matemática se contrapõe aquela que considera o conhecimento em constante construção e os indivíduos, no processo de interação social com o mundo, reelaboram, complementam e sistematizam os seus conhecimentos". Ensinar a resolver problemas requer que o professor coloque os alunos frente a diferentes situações. Ele deve encorajá-los a pensar por si mesmos, a levantarem suas próprias hipóteses, e as testá-las, a discutirem com seus colegas como e por que determinada estratégia pode ou não resolver o problema. Essa interpretação, de fato, parece ter sido desafiadora e resultou o efeito esperado de buscar outros elementos e raciocinar de forma distinta.

Após a apresentação do projeto, e aulas ministradas, foi colocado à disposição dos alunos o pós-teste, onde a média de acertos aumentou consideravelmente com relação ao pré-teste. Os alunos obtiveram médias de 73,53\% de acerto em questões de frações, $76,5 \%$ em questões de m.m.c. e 64,7\% de acerto em questões de equação do segundo grau. Ao serem perguntados se o projeto auxiliou no ensino aprendizagem e se a matemática visual é um bom recurso a ser usado em sala de aula obtivemos $100 \%$ de confirmação por parte dos alunos. Uma das alunas comentou:

O projeto matemático visual me ajudou muito a compreender e a entender muitos assuntos em que acreditava não compreender ou saber. A partir da iniciativa desse projeto, mudei minha forma de pensar e de como compreendia os assuntos de matemática, as aulas 
LIMA, Fabricio de Oliveira; BRANDÃO, Daniel Nicolau; SILVA, Douglas Muller; BRITO, Leonardo Rodrigues de; VIEIRA, Weverton de Barros

foram incríveis, com profissionais na área de matemática, que deram um show em compreensão e em explicação, sempre tendo e mantendo o bom humor, nos mostraram uma forma diferente de aprender matemática.

Além dos resultados, a matemática visual trabalha pela equidade em sala de aula. Quando uma aula se concentra em números, as diferenças de status entre alunos costumam aparecer. Mas se o conteúdo é visual, elas desaparecem e todos se sentem capazes de opinar. É possível acreditar que a matemática visual contribua para a equidade, ao valorizar formas diferentes de raciocínio, estimular engajamento e desenvolver níveis mais complexos de desempenho.

\section{CONSIDERAÇÕES FINAIS}

Mediante a pesquisa apresentada observamos que práticas educacionais que estimulem o desenvolvimento e a aprendizagem dos alunos são de extrema importância para a melhoria do ensino. Nessa perspectiva a partir da análise dos dados obtidos e afirmações realizadas pelos alunos, percebemos o quanto essa pesquisa é relevante e quantas aprendizagens significativas essa experiência proporcionou. Além de melhorar o desempenho dos alunos nos temas estudados, ainda mudou a relação que muitos tinha com a Matemática. É notório que a prática da matemática visual tende a ser parte integral da escola. Neste contexto vemos a importância de fugir dos padrões metodológicos meramente demonstrativos, despertando nos alunos habilidades, percepções sobre as diversas vertentes que a matemática pode culminar, estímulos das potencialidades individuais e busca da autonomia.

O uso da tecnologia, jogos, games, e metodologias que aproximem o aluno ao seu espaço é capaz de solucionar grande parte da dificuldade em aprender matemática na educação básica. Sendo a matemática uma linguagem que traduz a realidade, mostrando suas diferenças, percebemos a importância dos docentes ofertar diversas situações que tenham significados para os alunos, apresentando atividades que proporcionem diferentes formas de aprendizagem e possibilitando que o aluno instigue o pensar. Sendo assim, se faz necessário a aplicação de novas ferramentas educacionais que permitam que os alunos possam construir novos caminhos para aprendizagem. De fato, a matemática visual foi uma grande aliada para esses alunos, mostrando através dos resultados obtidos a grande evolução nas taxas de aprendizagem. 
LIMA, Fabricio de Oliveira; BRANDÃO, Daniel Nicolau; SILVA, Douglas Muller; BRITO, Leonardo Rodrigues de; VIEIRA, Weverton de Barros

Tendo considerado a importância da pesquisa para a educação, torna-se relevante o prosseguimento do estudo e aplicação da pesquisa no ensino médio, para que se tenha resultados a partir de aulas presenciais, rompendo assim paradigmas que afirmam que a matemática visual deve ser apenas usada para crianças. Também que os alunos possam criar soluções para problemas propostos utilizando a matemática visual, seria algo bastante interessante.

\section{REFERENCIAS}

1. Berch D. Making sense of number sense: implications for children with mathematical disabilities. J Learn Disabil. 2005;38(4):333-9.

2. BICUDO, M. A. V. (org.) Educação matemática. São Paulo: Moraes, 1985.

3. BITTAR, M. A abordagem instrumental para o estudo da integração da tecnologia na prática pedagógica do professor de matemática. Educar em Revista, Curitiba, Brasil, n. Especial 1/2011, p. 157-171, 2011. Editora UFPR

4. BRASIL. Ministério da Educação. Base Nacional Comum Curricular. Brasília, 2018.

5. BOALER, Jo. Metalidades Matemáticas: estimulando o potencial dos estudantes por meio da matemática criativa, das mensagens inspiradoras e do ensino inovador. Tradução de Daniel Bueno. Porto Alegre: Penso, 2018.

6. BOALER, JO et al (2016) Seeing as Understanding: The Importance of Visual

7. Mathematics for our Brain and Learning. J Appl Computat Math 5: 325. doi: 10.4172/2168-9679.1000325.

8. Boaler, J. \& Zoido, P. (in press). The Impact of Mathematics Learning Strategies upon Achievement: A Close Analysis of Pisa Data.

9. BECKER, Fernando. Epistemologia do Professor de Matemática. Petrópolis: Vozes, 2012.

10. CORSO, L. V. \& DORNELLES, B. V. Rev. Psicopedagogia 2010; 27(83): 298309

11. Cosenza, R. M., \& Guerra, L. B. (2011). Neurociência e educação: como o cérebro aprende. Porto Alegre: Artmed.

12. D’ AMBROZIO, Ubiratan. Da realidade a ação: Reflexões sobre a educação e matemática. Campinas: Unicamp, 1996.

13. D’AMBRÓSIO, U. A História da Matemática: questões historiográficas e políticas e reflexos na Educação Matemática. In: BICUDO, M. A. V. (org.). Pesquisa em Educação Matemática: concepções e perspectivas. São Paulo: UNESP, 1999, p. 97-115.

14. Doneda de Oliveira, V. S., \& Basniak, M. I. (202 1). FRAÇÕES E SUAS MÚLTIPLAS INTERPRETAÇÕES: reflexões sobre o ensino e a aprendizagem. Revista De História Da Educação Matemática, 7, 1-20. Recuperado de 
VISUAL MATHEMATICS FOR THE DEVELOPMENT OF MATHEMATICAL MENTALITIES IN STUDENTS OF A PUBLIC SCHOOL

LIMA, Fabricio de Oliveira; BRANDÃO, Daniel Nicolau; SILVA, Douglas Muller; BRITO, Leonardo Rodrigues de; VIEIRA, Weverton de Barros

<http://www.histemat.com.br/index.php/HISTEMAT/article/view/388> em 20/08/2021.

15. Feikes, D. \& Schwingendorf, K. (2008). The Importance of Compression in Children's Learning of Mathematics and Teacher's Learning to Teach Mathematics. Mediterranean Journal for Research in Mathematics Education 7 (2).

16. FERRARI, A. H. O Senso Numérico na Criança: Formação e Características. Doutorado em Educação Matemática. PUC-SP. São Paulo, 2008

17. FREIRE, Paulo. Pedagogia da autonomia: Saberes necessário a pratica educativa. São Paulo: Paz e Terra, 1996.

18. MENON, V. Arithmetic in child and adult brain. In: Handbook of mathematical cognition, K. R. Cohen \& A. Dowker (orgs.). Oxford University Press, 2014.

19. PARRA, C; SAIZ, I. (org.). Didática da matemática: reflexões psicopedagógicas. Porto Alegre: Artes médicas, 1996.

20. RAMOS, Daniela Karine; LORENSET, Caroline Chioquetta; PETRI, Giani. JOGOS EDUCACIONAIS: CONTRIBUIÇÕES DA NEUROCIÊNCIA À APRENDIZAGEM. Revista X, [S.1.], v. 2, n. 1.2016, nov. 2016. ISSN 19800614. Disponível em:

$<$ https://revistas.ufpr.br/revistax/article/view/46530/29523>. Acesso em: 05 set. 2021. doi:http://dx.doi.org/10.5380/rvx.v2i1.2016.46530.

21. 20. REVISTA EDUCAÇÃO. Conceito "Mentalidades Matemáticas" desfaz mitos com base na neurociência. Disponível em: https://www.revistaeducacao.com.br/mentalidades-matematicas/ . Acesso em: 24 de maio de 2019.

22. 21. Sampieri, R.H., Collado, C.F., \& Lucio, M. B. (2013). Metodologia de Pesquisa ( $5^{\mathrm{a}}$ ed.). Porto Alegre: Penso.

23. 22. Temple, C. (1997). Developmental cognitive neurpsychology. Hove (UK): Psychology Press. 